\title{
COVID and Post COVID Physical Therapy of a 63 year old Male with affection of Balance: A Case Report
}

\author{
Krishna Sukhadev Rathod*, Lakshmiprabha Deviprasad, Bhagyashree R Hajare, \\ Shreya M Chury, Saraswati K. Iyer.
}

P.T. School and Centre, Seth G.S. Medical College and K.E.M. Hospital, Maharashtra, India.

\section{ABSTRACT}

Background: The global outbreak of severe acute respiratory syndrome coronavirus 2 (SARS-CoV-2) disease 2019 (COVID-19) has a harsh effect throughout the world. A wide range of neuromuscular symptoms including myalgia, anosmia, loss of taste and muscle weakness have been seen post COVID-19 infection. Some of these symptoms may impair balance and activities of daily living. Balance is defined as ability of an individual to maintain the line of gravity within the base of support.

Objective: This case report study shows how physiotherapy management was helpful for a 63-year-old male during COVID and post-COVID rehabilitation to enhance his functional recovery. It also describes balance training strategies which were used in addition to regular pulmonary physiotherapy in a patient affected with COVID-19.

Case Report: This study reports a four month long physiotherapy protocol of a 63-year-old male patient admitted to a hospital due to COVID-19 in whom imbalance was also observed. Physiotherapy management was started for the same. The protocol included in-patient as well as out-patient exercises. Pulmonary physiotherapy along with neuro physiotherapy, in the form of balance training, were included. The patient showed good improvement in balance and regained his functional independence and hence discharged from physiotherapy treatment.

Conclusion: Thus, Covid and post- COVID-19 physiotherapy rehabilitation is essential to elicit positive adaptive changes to improve the functional capacity, independence in the daily activities and QOL.

KEY WORDS: COVID-19, Static Balance, Dynamic Balance, Fatigue, Neuromuscular Disorders.

Address for correspondence: : Krishna Sukhadev Rathod, P.T. School and Centre, Seth G.S. Medical College and K.E.M. Hospital, Maharashtra, India. E-Mail: krishsr1993@gmail.com

Access this Article online

Quick Response code

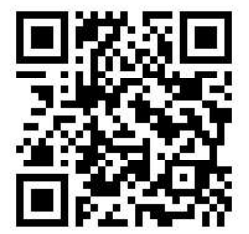

DOI: $10.16965 /$ ijpr.2021.200

Journal Information

International Journal of Physiotherapy and Research ISSN (E) 2321-1822 | ISSN (P) 2321-8975 https://www.ijmhr.org/ijpr.html DOI-Prefix: https://dx.doi.org/10.16965/ijpr

Article Information

Received: 25 Sep 2021

Peer Review: 25 Sep 2021

Revised: None
Accepted: 14 Nov 2021

Published (O): 11 Dec 2021

Published (P): 11 Dec 2021

\section{BACKGROUND}

The global outbreak of severe acute respiratory syndrome coronavirus 2 (SARS-CoV-2) disease 2019 (COVID-19) has a harsh effect throughout the world. SARS CoV-2 is a new disease that was first seen in December 2019 in China. It spread rapidly all over the world including India. The first case of COVID-19 in India was testified in January 2020 [1].

With the outbreak of coronavirus, the major area of concern was the respiratory system.
However, it has been established that coronavirus affection can extend beyond the respiratory system such as musculoskeletal system, cardiovascular system and nervous system. Symptoms of COVID 19 may vary, the most common symptoms being cough, fever, headache, dizziness, loss of smell and taste, fatigue along with hemiplegia, ischemic heart disease etc.

A chronic post-viral affection characterized by fatigue, muscle pain and sleep disturbances 
has previously been reported following SARS coronavirus infection, which emerged from South East Asia in early 2003 [2,3].

A wide range of neuromuscular symptoms include myalgia, anosmia, loss of taste and muscle weakness. Some of these symptoms may impair balance and activities of daily living [2].

Post covid complications: Common signs and symptoms which are seen for a long time include: Fatigue, difficulty in breathing or breathlessness, Cough, Joint pain, Chest pain, Memory, Lack of concentration or Sleep problems, Pain in muscles or Headache, Increased heart rate, Loss of smell or taste, Depression or anxiety, Fever and Dizziness.

Various organs may get damaged due to COVID19 and this increases the risk of long-term health problems. The following organs may get affected by COVID-19:

Heart: Various imaging tests done months after recovery from COVID-19 have shown lasting damage to the heart, even in people who have experienced only mild COVID-19 symptoms. This increases the risk heart complications like heart failure in the future.

Lungs: The tiny air sacs (alveoli) of the lung undergo long lasting damage due to pneumonia associated with COVID-19. The result of which is scar tissue formation which can lead to long-term breathing problems.

Brain: There is an increased risk of stroke, seizures and Guillain-Barre syndrome (a condition that causes temporary paralysis) even in young adults affected with COVID-19.

Some adults and children may get exposed to multisystem inflammatory syndrome after they have had COVID-19. In this condition there may be inflammation of some organs and tissues [313].

Balance is defined as ability of an individual to maintain the line of gravity within the Base of support. Somatosensory / Proprioceptive System, Vestibular System and Visual System provide input regarding the body's equilibrium to the Central Nervous System. The feedback sent by these three systems about the body's orientation to The Central Nervous System is integrated and subsequently a corrective, stabilizing torque is generated by selectively activating muscles Int J Physiother Res 2021;9(6):4079-86. ISSN 2321-1822 for maintaining balance [4].

Static Balance: Static Balance is the capability of the body to maintain itself in some fixed posture [9]. It is when the body is able to maintain postural stability and orientation with centre of mass over the base of support at rest.

Single Leg Stance test (SLS) is a feasible, routine static balance assessment tool in Neuro Physiotherapy for measuring risk of fall and consequently assessing neurological and musculoskeletal status in static balance control and best of three score for the SLS measured in seconds.

Dynamic Balance: Dynamic balance is the ability to maintain postural stability and orientation with centre of mass over the base of support while the body parts are in motion [10].

Functional Reach Test (FRT) is an easy, practical, reliable, valid, routine clinical outcome measure for determining dynamic balance in one simple task. Difference between the start and end position which is the reach distance is measured in centimetres and best of three score calculated.

Fatigue: Fatigue is a term used to describe an overall feeling of tiredness, lack of energy, lack of motivation. It is different from feeling drowsy or sleepy. However, being sleepy may be a symptom of fatigue. Many mild to serious medical conditions may have it as a common symptom. However, lack of exercises, poor diet and other lifestyle choices may also result in fatigue. Because the symptom of fatigue is often vague, clinical evaluation requires the consideration of various physical and psychological precipitating factors.

One of the studies gives us insight about post COVID-19 fatigue syndrome, a long-term sequela of COVID-19 which is similar to myalgia encephalomyelitis / chronic fatigue syndrome in COVID-19 patients. There is reduced outflow of cerebrospinal fluid through the cribriform plate, further leading to congestion of the glymphatic system due to damage to olfactory sensory neurons. This causes build-up of toxic substances within the central nervous system [11].

The Fatigue Numerical Rating Scale (NRS), is a patient-administered, 11-point horizontal scale from 0 to 10 , with 0 representing 'no fatigue' 
and 10 representing 'as bad as you can imagine'. It is a very feasible, easy to administer during routine Physiotherapy assessments. It gives us a patient's perspective in understanding Fatigue experienced due to COVID -19 and not pertaining to exercises.

\section{CASE REPORT}

A 63-year-old male patient was admitted in private hospital for 33 days with covid positive status, and symptoms of fever, cough, dyspnea. Initially he was on Non-Invasive Ventilation for 21 days then shifted on $\mathrm{O}_{2}$ with Face Mask. $\mathrm{He}$ was detected with pulmonary thromboembolism, Diabetes Mellitus and Hypertension when he was admitted to the hospital and was immobilized for the same. Due to financial issues, he was shifted to government tertiary health care center with Stage II of the disease and a CT score of $14 / 25$. He was symptomatic with fever, dry cough, loss of smell, breathing difficulty, swelling in his feet, Dizziness, Fatigue, weakness, and loss of balance. Initially he was on $\mathrm{O}_{2}$ with Face Mask for 6 days and then shifted on Nasal Prongs with 2 lit $\mathrm{O}_{2}$ and maintained saturation. Repeat HRCT was done after 11 days of admission to tertiary care centre and it was suggestive of late stage of atypical pneumonia (CORAD-6), CT severity-moderate, No pulmonary thromboembolism. Since he was maintaining saturation 02 was reduced to 1 lit and subsequently was off 02 . He was discharged after one month stay in the tertiary care center after 2 RTPCRs showed negative report. His tenure of in-patient stay was for almost 2 months totally. As soon as the patient was admitted to Tertiary care center, Physiotherapy sessions were started. (The earlier data about private set up was unavailable.)

Physiotherapy session began with patient education followed by positioning, CARP protocol, breathing control, thoracic mobility exercise, bed/bedside exercises, gradual progression from supine to sitting to standing to walking and Limb elevation for swelling. Pre and post HR and $\mathrm{O}_{2}$ Sat were monitored during every session and exercise intensity was decided accordingly. Sufficient rest pauses were given initially when he was not maintaining saturation. When the patient started standing/ walking imbalance was observed and FRT, SLS were recorded for balance and NRS were assessed for fatigue.
On that day his FRT was $29.5 \mathrm{~cm}$ which was below normative value for Indian males in the age group of 61 to 70 years [12], SLS was 3s which indicates static balance affection and NRS was 4 . On the day of discharge, he walked 10meter distance with support.

Physiotherapy follow up was done from December to March at his residence. Post Covid rehabilitation was done every day for one and half hours with adequate rest pauses because his saturation was dropping sometime but maintained with rest.

At end of each session crepe bandaging was done in pattern of eight and positioning was given for the swelling in the feet followed by elevation and ankle toe movements.

In Respiratory physical therapy, CARP protocol, Purse lip breathing, segmental breathing exercises, incentive spirometry, chest PNF, and chest expansion exercises were given. In every session pre-post SPO2 and HR were assessed. Progression of these exercises were done with the increasing number of repetition and sets according to patient's responses and compliance over the next three months tenure. The strategies used for Balance Training with progression were Reach outs in uniplanar to multiplanar dimensions, Wide base of support initially to narrow base of support, eyes open initially to eyes closed and use of appropriate feedback along with the above exercises.

Balance and Mobility training started with training for improving sitting balance with reach outs and arm movements $\rightarrow$ sit to stand training (with and without support) $\rightarrow$ to standing initially from wide base of support to narrow base of support $\rightarrow>$ Tandem standing (with eyes open and eyes closed)à one leg standing (with eyes open and eyes closed) $\rightarrow$ level walking (with and without support) $\rightarrow$ step up step down $\rightarrow$ stair climbing. The Intensity, frequency and duration of exercises were modified and progressed gradually over the 3 months of post covid rehabilitation.

Strength training exercises were given for $B / L$ $\mathrm{LL}$ and $U \mathrm{~L}$ with theraband and progression was done by gradually increasing the resistance and no. of sets of exercise.

Visual and auditory cuing were used to give 
appropriate feedback during the session. Feedbacks were given to the patients in the form knowledge of results and knowledge of performance during and after each session. The patient was motivated to perform the best of his abilities in each session.

LL Swelling management: Limb elevation (pillows under the LL) with ATM during hospital stay, Crepe bandaging with ATM in limb elevated position was done at the end of every session till the oedema resolved by end of February.

During the entire session SPO2 and HR was regularly monitored. The intensity and duration of exercise were modified according to Spo 2 and $H R$ values and Fatiguability of the patient. Sufficient rest pauses were incorporated as required.

Monthly evaluation of FRT, SLS for Balance and pertaining to exercise sessions) were done.

\section{Post COVID Rehab sessions}

Table 1: Physiotherapy management protocol for the patient.

\begin{tabular}{|c|c|c|c|c|c|c|c|c|c|c|c|}
\hline & December & & Janu & iary & & & Febr & uary & & $\mathrm{M}_{\mathrm{z}}$ & rch \\
\hline PT sessions & 4th week & 1st week & 2nd week & 3rd week & 4th week & 1st week & 2nd week & 3rd week & 4th week & 1st week & 2nd week \\
\hline Incentive spiro & $v$ & $\mathrm{v}$ & $\mathrm{v}$ & $\mathrm{V}$ & $\mathrm{v}$ & $\mathrm{v}$ & $\mathrm{v}$ & $\mathrm{v}$ & $\mathrm{v}$ & $\mathrm{v}$ & $\mathrm{v}$ \\
\hline Bed/Bedside mobility & $v$ & $v$ & $v$ & $v$ & $\mathrm{v}$ & $v$ & $v$ & 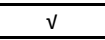 & $v$ & $v$ & $\mathrm{v}$ \\
\hline Purse lip breathing & $v$ & $v$ & $v$ & $v$ & 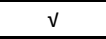 & $v$ & $v$ & $v$ & $\mathrm{v}$ & v & $\mathrm{v}$ \\
\hline $\begin{array}{l}\text { Segmental breathing } \\
\text { exercise }\end{array}$ & $v$ & $v$ & $v$ & $v$ & $v$ & $v$ & $v$ & $v$ & $v$ & $v$ & v \\
\hline $\begin{array}{l}\text { Thoracic expansion } \\
\text { exercise }\end{array}$ & $v$ & $v$ & $v$ & $v$ & $v$ & $v$ & $v$ & $v$ & $v$ & $v$ & $v$ \\
\hline \begin{tabular}{|l|}
$\begin{array}{l}\text { Postural correction } \\
\text { exercise }\end{array}$ \\
\end{tabular} & $v$ & $v$ & $v$ & $v$ & $v$ & $v$ & $v$ & $v$ & $v$ & $v$ & $v$ \\
\hline $\begin{array}{l}\text { TheraBand exercise } \\
\text { (with progressive } \\
\text { resistance) }\end{array}$ & & $v$ & $v$ & $v$ & $v$ & $v$ & $v$ & $v$ & $v$ & $v$ & $v$ \\
\hline Sit to stand with support & $v$ & $v$ & $v$ & $v$ & & & & & & & \\
\hline $\begin{array}{l}\text { Sit to stand without } \\
\text { support }\end{array}$ & & & & $v$ & $v$ & $v$ & $v$ & $v$ & $v$ & $v$ & $v$ \\
\hline $\begin{array}{l}\text { Standing with broad BOS } \\
\text { (as per patient's } \\
\text { comfort) }\end{array}$ & $v$ & $v$ & $v$ & $v$ & $v$ & & & & & & \\
\hline $\begin{array}{l}\text { Standing with narrow } \\
\text { BOS }\end{array}$ & & & & $v$ & $v$ & $v$ & $v$ & $v$ & $v$ & $v$ & $v$ \\
\hline $\begin{array}{l}\text { Tandem standing with } \\
\text { eyes open }\end{array}$ & & & & & $v$ & $v$ & $v$ & $v$ & & & \\
\hline $\begin{array}{l}\text { Tandem standing with } \\
\text { eyes closed }\end{array}$ & & & & & & & $v$ & $v$ & $v$ & $v$ & $v$ \\
\hline Reach outs & 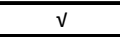 & $\mathrm{v}$ & 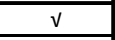 & 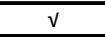 & 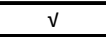 & $\sqrt{ }$ & 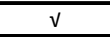 & 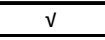 & $\sqrt{ }$ & & \\
\hline $\begin{array}{l}\begin{array}{l}\text { One leg standing with } \\
\text { support eyes open }\end{array} \\
\end{array}$ & & & & & $v$ & $v$ & $v$ & $v$ & $v$ & & \\
\hline $\begin{array}{l}\text { One leg standing without } \\
\text { support eyes open }\end{array}$ & & & & & & & $v$ & $v$ & $v$ & $v$ & $v$ \\
\hline $\begin{array}{l}\text { One leg standing without } \\
\text { support eyes closed }\end{array}$ & & & & & & & $v$ & $v$ & $v$ & $v$ & $v$ \\
\hline $\begin{array}{l}\text { Walking with support } \\
\text { (progressively increased } \\
\text { duration of walking) }\end{array}$ & $v$ & $v$ & $v$ & $v$ & & & & & & & \\
\hline $\begin{array}{l}\text { Walking without support } \\
\text { (progressively increased } \\
\text { duration of walking) }\end{array}$ & & & & $v$ & $v$ & $v$ & $v$ & $v$ & $v$ & $v$ & $v$ \\
\hline Tandem walking & & & & & & 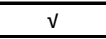 & $v$ & $\sqrt{ }$ & $\sqrt{ }$ & $\mathrm{v}$ & $v$ \\
\hline $\begin{array}{l}\text { Step up, Step down with } \\
\text { support }\end{array}$ & & & & $v$ & $v$ & $v$ & $v$ & & & & \\
\hline \begin{tabular}{|l|}
$\begin{array}{l}\text { Step up, Step down } \\
\text { without support }\end{array}$ \\
\end{tabular} & & & & & & $v$ & $v$ & $v$ & $v$ & & \\
\hline $\begin{array}{l}\text { Stair climbing with } \\
\text { support }\end{array}$ & & & & & & $v$ & $v$ & $v$ & & & \\
\hline $\begin{array}{l}\text { Stair climbing without } \\
\text { support }\end{array}$ & & & & & & & & & $v$ & $v$ & $v$ \\
\hline $\begin{array}{l}\text { LL swelling management } \\
\text { (Limb elevation, ATM } \\
\text { and Crepe bandage) }\end{array}$ & $v$ & $v$ & $v$ & $v$ & $v$ & $v$ & $v$ & $v$ & $v$ & & \\
\hline
\end{tabular}


Krishna Sukhadev Rathod et al., COVID and Post COVID Physical Therapy of a 63 year old male with affection of Balance: A Case Report.

Table 2: Assessment of Balance and Fatigue.

\begin{tabular}{lcccc}
\hline & December & January & February & March \\
\hline $\begin{array}{l}\text { Single leg } \\
\text { stance test }\end{array}$ & 3 second & 5 second & 30 second & 108 seconds \\
$\begin{array}{l}\text { Forward reach } \\
\text { test distance }\end{array}$ & $29.5 \mathrm{~cm}$ & $31 \mathrm{~cm}$ & $32 \mathrm{~cm}$ & $34 \mathrm{~cm}$ \\
NRS for fatigue & 4 & 2 & 0 & 0
\end{tabular}

Inference: Table no-2 shows that Single leg stance time increased from 3 seconds to 108 seconds, Forward Reach Test distance increased from $29.5 \mathrm{~cm}$ to $34 \mathrm{~cm}$ and NRS for fatigue reduced from 4 to 0 during post-COVID rehabilitation.

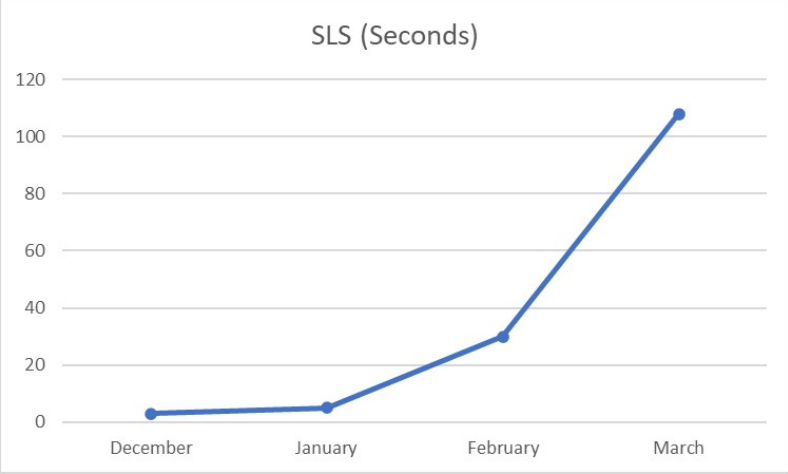

Graph 1: Graph showing improvement of SLS scores during post-COVID physiotherapy.

\section{RESULT AND DISCUSSION}

The table no. 2 shows that there was an improvement in the static and dynamic balance performance every month indicated by increase in SLS time and FRT distance with regular post Covid19 Physical Therapy. Overall Fatigue was also reduced to zero with regular exercise. These effects could be attributed to the various approaches of Physical Therapy interventions given to this patient. Covid-19 patient needed long term complex rehabilitation as it presented with a mixture of cardio-respiratory, neuromuscular and mental health problems [16].

Patient's education in Covid-19 helps patient to be motivated to do exercise, understand about their condition and to understand the scope of recovery. In physiotherapy, recently attention is being given to patient education (Norton, 1986; Leathley, 1988; Hayne, 1988). It is recognized that patient education can contribute to patient compliance and recall and this may, in turn, enhance the effects of treatment (Croft, 1980; Wagstaff, 1982; May, 1983; Ice, 1985). This may facilitate patient compliance with the regimens prescribed [20].
FRT (cms)

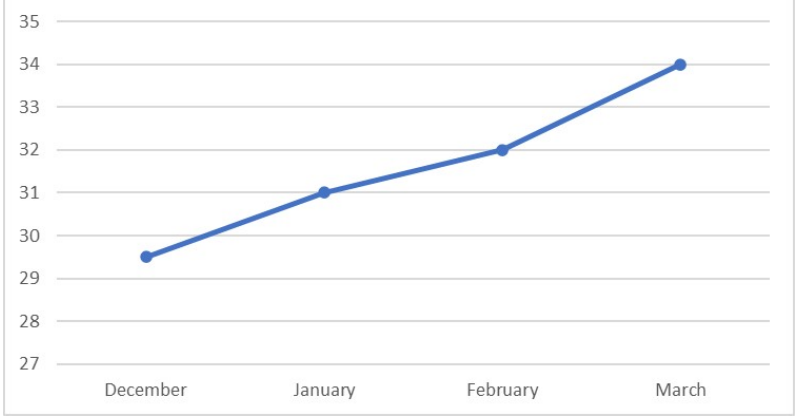

Graph 2: Graph showing improvement of FRT distance during post-COVID physiotherapy.

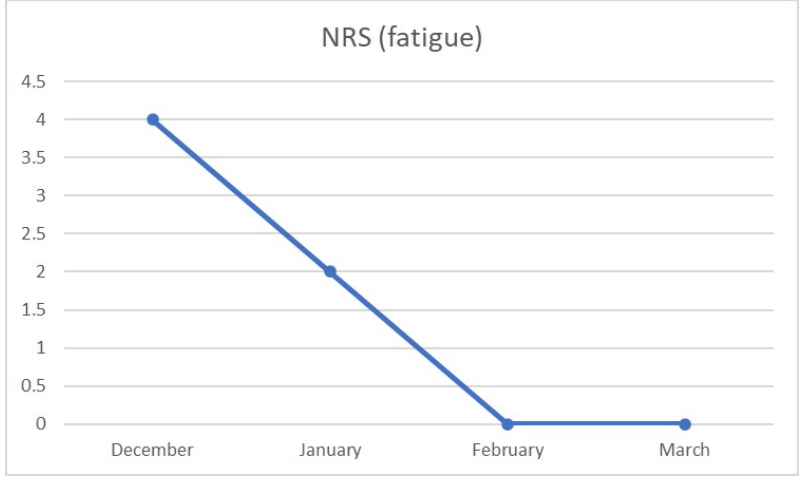

Graph 3: Graph showing reduction in NRS for fatigue during post-COVID physiotherapy.

The systematic scoping review (March 2020) determined the impact of bed rest on balance control and the sensorimotor systems among healthy adults [9]. Early rehabilitation has beneficial effects on patients as it reduces the adverse effects of immobility, improving respiratory function, cardiovascular conditioning, level of consciousness, functional independence, and psychological well-being. It has been reported in systematic reviews for reduction of hospitalization length of stay, thereby favouring better functional capacity on a long-term basis and quality of life after discharge $[14,15]$.

Due to the systemic effect of the post-COVID19 syndrome, a systematic and multifactorial evaluation of the physical and functional capacity is needed to facilitate the prescription of tailored exercise programs to enable a complete functional recovery and a return to pre functional level post Covid-19. Individually tailored exercise prescriptions were planned and given as per the need and requirement of the patient. Breathing exercise helped to improve the lung expansion, improve ventilation in lungs and helped to maintain ventilation perfusion ratio and improved saturation. Breathing exercises slows the rate of breathing also improving 
breathing control and thus expends less energy. Breathing exercise also causes relaxation, lowering the harmful effects of the stress hormone cortisol on the body.

The mechanisms involved in balance were summarized by Bannister [9]. He noted sufficient power is required in muscles of lower limb and trunk in normal standing to maintain the body erect and in dynamic balance to maintain movement and stability. Strength training helped to improve balance control and improve physical functions. Strength training exercise programs have been extensively studied and they are safe and effective among vulnerable people in reversing frailty and weakness and reinstating functional capacity in the short- and long-term rehabilitation. Indeed, strength training bestows unique benefits to the musculoskeletal system, improving both morphological (increased number of sarcomeres, increased synthesis of contractile assemblies of actin and myosin and altering muscle fibres' composition) and neural (boosting neurological system and intermuscular coordination) factors and modulating the whole-body metabolism $[17,19]$ Strengthening and balance control have also showed to improve the walking speed.

Balance training exercises given in this study, challenge the visual and proprioceptive sensory system and neuro musculoskeletal system through changes in direction, change in position of the center of mass during static and dynamic exercise. It is found to improve static and dynamic balance and quality of life [21]. The improvement seen in postural control in old people due to balance training is well documented. Balance training may decrease activation of antagonist muscle, shorten onset latency of muscle activation, augment reflex activity, increase maximal force production capacity, increase the length of recovery steps to external perturbation [22]. They also help to strengthen the muscles in the thighs and the muscles in the centre of the body which gives stability (core muscles) which help to maintain balance and reduces risk of fall [23].

Crepe bandaging helped to improve the venous return and reduced swelling by draining extracellular fluid.
Post-COVID-19 syndrome is one of the increasingly common challenge as the pandemic has evolved. The aim of physiotherapy treatment was to enhance general fitness as well as to solve other range of problems such as fatigue, emotional disturbance, lack of confidence, and performance of effortful activities such as walking, as it was seen to be limited. Thus, many COVID-19 patients need local, community-based rehabilitation services particularly due to restrictive situations (such as lockdown periods) or people with severe mobility limitations $[17,18]$. Physical fitness status can be a regulating modifiable factor for the improvement in metabolic and functional adaptations of T-lymphocytes and monocytes, counteracting inflammatory environments caused by expanded adipose tissue and sedentary behaviour, also as delaying the immunosenescence caused by aging. Regular release of muscle-derived anti-inflammatory cytokines (IL-6, IL-7, IL-10, IL-15), along with the inhibition of pro-inflammatory cytokines (IL- $1 \alpha$, IL-18, TNF- $\beta$ ), have been claimed to play important roles in the beneficial effects of exercise on immunity. Exercise weakens immuno-senescence by maintaining the peripheral T-cell pool and natural killer cell compartments, and it seems to improve the immune response to SARSCoV-2 antigens. Exercises have shown to be beneficial in numerous pathologies with which the post-COVID-19 syndrome shows resemblance both in terms of symptoms and its possible pathogenic mechanisms. It is worth considering the potential favourable effect of exercises for recovery of the Covid-19 patients.

Research shows that regular exercises are a powerful tool in enhancing the quality of life, in managing mood swings and its illnesses (depression and anxiety), in attenuating psychological stress and in modulating the perception of pain. Exercise helps to change the brain structure and function to stimulate a healthier neurological phenotype [19].

\section{CONCLUSION}

With improved FRT, SLS and NRS values, patient showed good recovery and regained his functional independence. His exercise capacity also increased. Thus, patient was discharged from Physiotherapy after a duration of almost 
5 months ( 1 Month of COVID rehab and 4 months of post COVID rehab) from the onset of the disease. Thus, COVID-19 and post COVID19 physiotherapy rehabilitation is essential to elicit positive adaptive changes to improve the functional capacity, independence in the daily activities and QOL.

\section{ACKNOWLEDGEMENTS}

We would like to acknowledge our patient for his co-operation and time throughout the treatment procedure. We would also like to express our heartfelt gratitude to Dr. Mariya Jiandani (Associate Professor in physiotherapy) who is the Physiotherapy in-charge of COVID-19 In-patients.

\section{ABBREVIATIONS}

SARS CoV-2: severe acute respiratory syndrome coronavirus 2

Covid-19: Coronavirus disease

SLS: single leg stance

FRT: functional reach test

BOS: base of support

QOL: quality of life

NRS- Numerical Rating scale

UL- Upper limb

LL- lower limb

ATM- Ankle toe movements

\section{Conflicts of interest: None}

\section{REFERENCES}

[1]. Dipti Baban Geete, Jaimala Vijay Shetye, and Anil Manohar Sathe, Physiotherapy management of geriatric COVID-19 patients in an intensive care unit of a government tertiary care hospital: A case series: World Journal of Advanced Research and Reviews

[2]. Abdullahi A, Candan SA, Abba MA, Bello AH, Alshehri $M A$, Afamefuna Victor E, Umar NA, Kundakci B. Neurological and musculoskeletal features of COVID-19: a systematic review and meta-analysis. Frontiers in neurology. 2020 Jun 26;11:687.

[3]. Moldofsky H, Patcai J. Chronic widespread musculoskeletal pain, fatigue, depression and disordered sleep in chronic post-SARS syndrome; a case-controlled study. BMC Neurol 2011;11(1). https:// doi.org/10.1186/1471-2377-11-37

[4]. Peterka RJ. Sensorimotor integration in human postural control. J Neurophysiol 2002;88:1097-1118.

[5]. Cynthia Lions, Emmanuel Bui Quoc,Sylvette WienerVacher,and Maria P. Bucci1:Postural control in strabismic children: importance of proprioceptive information:Front Physiol. 2014; 5: 156.
[6]. Hwang S, Agada P, Kiemel T, Jeka JJ (2014) Dynamic Reweighting of Three Modalities for Sensor Fusion. PLoS ONE 9(1): e88132. doi:10.1371/ journal.pone.0088132.

[7]. Friedrich M, Grein HJ, Wicher C, Schuetze J, Mueller A, Lauenroth A, Hottenrott K, Schwesig R: Influence of pathologic and simulated visual dysfunctions on the postural system.:Exp Brain Res. 2008 Mar; 186(2):305-14.

[8]. Nicoleta Bugnariu and Joyce Fung;Aging and selective sensorimotor strategies in the regulation of upright balance:J Neuroengineering Rehabil. 2007; 4: 19

[9]. Bannister R: Brain's Clinical Neurology, ed 3. New York, NY,Oxford University Press, Inc, 1969;51-54, 102

[10]. GOLDIE PA, BACH TM, EVANS OM. Force platform measures for evaluating postural control: fckLRReliability and validity. Arch Phys Med Rehabil. 1989; 70:510-517

[11]. Wostyn P. COVID-19 and chronic fatigue syndrome: Is the worst yet to come? Med Hypotheses. 2021 Jan;146:110469.

[12]. Dani VB, Shah R, Sheth R. Functional reach test: Establishing the reference value in healthy adults of Gujarat, India. Acta Med Int [serial online] 2019 [cited 2021 Aug 19];6:89-92.

[13]. COVID-19 (coronavirus): Long-term effects

[14]. Udina, C., Ars, J., Morandi, A. et al. Rehabilitation in Adult Post-COVID-19 Patients in Post-Acute Care with Therapeutic Exercise. J Frailty Aging 2021;10:297-300. https://doi.org/10.14283/ jfa.2021.1

[15]. "Effect of Early Mobilization on Respiratory and Limb Muscle Strength and Functionality of Nonintubated Patients in Critical Care: A Feasibility Trial, Critical Care Research and Practice, 2020, Article ID 3526730, 9 pages, 2020. https://doi.org/ $10.1155 / 2020 / 3526730$

[16]. Zhu, Yuetong et al. Summary of respiratory rehabilitation and physical therapy guidelines for patients with COVID-19 based on recommendations of World Confederation for Physical Therapy and National Association of Physical Therapy. Journal of physical therapy science 2020;32(8):545549. doi:10.1589/jpts.32.545.

[17]. Jimeno-Almazán,A.; Pallarés, J.G.; Buendía-Romero, Á.; Martínez-Cava, A.; Franco-López, F.; SánchezAlcaraz Martínez, B.J.; Bernal-Morel, E.; CourelIbáñez, J. Post-COVID-19 Syndrome and the Potential Benefits of Exercise. Int. J. Environ. Res. Public Health 2021;18:5329. https://doi.org/10.3390/ ijerph18105329.

[18]. Peter Thomas, et al, Physiotherapy management for COVID-19 in the acute hospital setting: clinical practice recommendations, Journal of Physiotherapy, 2020;66(2):73-82.

[19]. Wade DT. Rehabilitation after COVID-19: an evidence-based approach. Clin Med (Lond). 2020;20(4):359-365. doi:10.7861/clinmed.20200353 
[20]. Emmy M Sluijs, Patient Education in Physiotherapy: Towards a planned approach, Physiotherapy 1991;77(7):503-508. ISSN 0031-9406,https:// doi.org/10.1016/S0031-9406(10)61855-X.

[21]. Dunsky A. The effect of balance and coordination exercises on quality of life in older adults: a minireview. Frontiers in aging neuroscience. 2019 Nov 15;11:318.

[22]. Wiesmeier IK, Dalin D, Wehrle A, Granacher U, Muehlbauer T, Dietterle J, Weiller C, Gollhofer A, Maurer $C$. Balance training enhances vestibular function and reduces overactive proprioceptive feedback in elderly. Frontiers in aging neuroscience. 2017 Aug 11;9:273.

[23]. Regterschot GR, Folkersma M, Zhang W, Baldus $H$, Stevens M, Zijlstra W. Sensitivity of sensor-based sit-to-stand peak power to the effects of training leg strength, leg power and balance in older adults. Gait \& posture. 2014 Jan 1;39(1):303-7.

How to cite this article: Krishna Sukhadev Rathod, Lakshmiprabha Deviprasad, Bhagyashree R Hajare, Shreya M Chury, Saraswati K. Iyer. COVID and Post COVID Physical Therapy of a 63 year old male with affection of Balance: A Case Report. Int J Physiother Res 2021;9(6):4079-4086. DOI: $10.16965 /$ ijpr.2021.200 